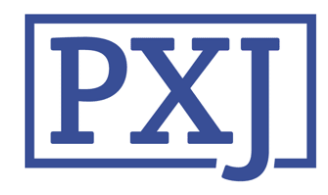

Patient Experience Journal

Volume 7

Issue 2 Special Issue: Sustaining a Focus on

Human Experience in the Face of COVID-19

Article 16

2020

\title{
How University of Chicago Medicine designed virtual rounding to maintain human connections during COVID-19
}

Susan M. Murphy

University of Chicago Medicine

Follow this and additional works at: https://pxjournal.org/journal

Part of the Health and Medical Administration Commons, Health Policy Commons, Health Services Administration Commons, and the Health Services Research Commons

\section{Recommended Citation}

Murphy SM. How University of Chicago Medicine designed virtual rounding to maintain human connections during COVID-19. Patient Experience Journal. 2020; 7(2):80-82. doi: 10.35680/ 2372-0247.1457.

This Case Study is brought to you for free and open access by Patient Experience Journal. It has been accepted for inclusion in Patient Experience Journal by an authorized editor of Patient Experience Journal. 


\section{How University of Chicago Medicine designed virtual rounding to maintain human connections during COVID-19}

\section{Cover Page Footnote}

This article is associated with the Innovation \& Technology lens of The Beryl Institute Experience Framework. (http://bit.ly/ExperienceFramework). You can access other resources related to this lens including additional PXJ articles here: http://bit.ly/PX_InnovTech 


\title{
How University of Chicago Medicine designed virtual rounding to maintain human connections during COVID-19 \\ Susan M. Murphy, University of Chicago Medicine,susan.murpby@uchospitals.edu
}

\begin{abstract}
This story is about how the Patient Experience and Engagement Program (PEEP) team at University of Chicago Medicine adapted our patient rounding program from in-person to virtual during the COVID-19 crisis. With creativity and ingenuity, our team shifted on a dime to ensure that patients received the kind of care and connection that allowed them to start the recovery process from what could otherwise be a traumatizing experience before they even leave the hospital. I will explain the steps we took to quickly transition our rounding program from in-person to virtual. But more than that, I want to convey the power of connection to support healing - especially when circumstances are challenging. During and after the COVID-19 pandemic, people need ways to maintain human connections.
\end{abstract}

\section{Keywords}

COVID-19, virtual rounding, human connection, patient experience

\section{There is Healing Power in Humanity}

I have been a nurse for more than 30 years and caring for patients has been the great privilege of my life. In my role as the Chief Experience Officer at the University of Chicago Medicine, I've worked hard to build a team of people who put heart and compassion at the core of everything they do. We see it as our mission to support every team member at UChicago and help them provide the best possible care for our patients.

When the COVID-19 crisis struck Chicago, I was asked to consider how to continue providing support for our care teams and patients while limiting face to face patient care interactions. Meaning, our care in the patient rooms had to be limited to specific clinical care needs. Our teams needed to feel confident in the proper PPE requirements for this type of unknown virus. Thus, in real time, we pivoted our approach to include an Observation Role on the inpatient units and a movement from in-person care rounds to virtual care connections.

I was asked to step out of my ordinary leadership role and put my clinical hat back on. I spent a day outside a COVID-19 unit as an observer, helping care team members don and doff their PPE safely and providing them with moral support and compassion as they went in for what would prove to be the hardest shifts in their careers.

I didn't know at the outset that these shifts would turn out to be some of the hardest of my career. As a nurse who has always believed in the power of human connection, it broke my heart to see patients isolated in their rooms with no loved ones, no visitors. I knew our clinical teams were providing them the best possible care, but I just worried that patients were lonely. I couldn't bear the weight of imagining their fear. I worried that the mental struggle would add to the burden of their recovery.

\section{A Vital Change was Needed}

After one of my shifts, I shared my concerns with leaders on the PEEP team. I just couldn't carry my concerns by myself. As a result of COVID, we no longer were able to connect with our patients through in-person rounding practices. My observations and understanding the importance of connection to healing, particularly during this time, required a vital change. We had to find a new way to be with our patients. Fortunately, the members of this team are creative, resilient and incredibly caring. They jumped to a solution.

For quite a few years, we have had a leader rounding program at UChicago in which nursing leaders and PEEP team members visit patients every day to check on their comfort, their care and their concerns. We capture feedback and track follow-ups in our rounding platform, which helps us ensure we close the loop on patient needs, forward recognition to team leaders, and analyze data for systemic issues that need to be addressed through process improvement.

By necessity, we halted our bedside rounding program when preparations for COVID-19 care began in earnest. Safety was our top priority. And it wasn't safe to have anyone who didn't have a direct role in clinical patient care enter the patient's room.

Patient Experience Journal, Volume 7, Issue 2 - Special Issue: Sustaining a Focus on Human Experience in the Face of COVID-19 


\section{Creating New Processes \& Practices}

But what if we didn't have to enter the patient's room? What if we could call them instead? Would that help to alleviate the loneliness and isolation that patients might be experiencing under our new and necessary COVID-19 restrictions? Would that help ease the burden on staff members who are also worried about patient well-being, but struggling to manage their own stress and well-being with fears of contamination and new protocols and PPE requirements? Those are the questions my team leaders asked. Then, within three days, they designed and implemented a program that would allow us to do just that.

The first questions we faced were logistical. How could we get the right phone numbers for the right patients? How could we coordinate with care teams so our calls would be helpful and not disrupt patient care? We reached out to our call center team to make sure we had a correct listing of every room and the corresponding phone number. They shared that with us, and then we reached out to the operational leaders on each unit.

Prior to COVID-19, our PEEP team members have always regularly coordinated with unit managers to help understand their unit's unique needs, resources and culture and to provide relevant support for experience improvement efforts. They have become extensions of the leadership teams on the units they serve. So, PEEP team members reached out to their respective unit leaders and let them know of our plans to shift to virtual rounds. The leaders were grateful to have additional support for their patients and asked only that we immediately escalate any urgent clinical issues to their attention via a phone call as well as through our automated process.

\section{Technology Helps Make Meaningful Connections}

The next question was about patient conversations. What should we talk about with patients? Historically, we've ask about their diagnosis, whether they understand their care, and if they have any clinical or comfort needs. But these calls felt different. These were less about care and diagnosis and more about connecting as human beings. We wanted the call to be a highlight in each patient's day. We wanted to create a feeling of spaciousness and humanity.

So, we built a new call plan. It's less of a script and grounded in the core principles from our hArt of Medicine ${ }^{\circledR}$ program that focuses on empathic interactions, therapeutic relationships and meaningful connections to create a culture of caring. We asked our PEEP callers to create a patient centric conversation, where the patient would experience feeling cared about vs. the care delivered. We reminded them to listen with the intention to understand and to not interrupt. We reinforced the impact of repeating back the patient's words, and summarizing what was discussed, validating understanding and bringing the patient's voice further into the conversation.

We worked with our technology partners to adapt our care rounding templates to reflect these prompts and to rethink some of our follow-up processes. If patients had needs from our environmental services or food services teams, we used the built-in process management tools within the rounding solution to make sure requests were routed appropriately and that we closed the loop on patient needs. But, as requested, if we uncovered clinical needs, our standard work included a phone call from the rounder to the nurse leader on the unit. This would not only allow them to respond quickly, it would also provide a human touch and help the nurse leader balance the workload for nurses who are doing so much to care for our patients in difficult circumstances.

The next question was about alignment. Who would our team members call and how often would we call them? We decided that patients needed our support and a human connection every day. So, we staffed the call plan seven days a week. We also ask our patients at the close of every call if they would like another call the next day. If they answer yes, we make sure that the same PEEP team member calls every day during that patient's hospital stay. If a patient is in the hospital for ten days and is able to speak on the phone during the entire stay, he will receive a call from the same person each day. This consistency lets our team members build meaningful connections and provide the kind of caring that we believe allows the patient's recovery to start before they ever leave the hospital.

Upwards of $90 \%$ of the patients we call tell us they would like another call the following day. Patients have told us that they look forward to these calls as a bright spot in their day. That's the driving force for us to continue the calls until we can go back to in-person rounding.

\section{Patients and Staff Share Stories of Compassion}

While our patient experience outcomes rose to the occasion and demonstrated top box outcomes of "Likelihood to Recommend" which landed us in the $76^{\text {th }}$ percentile, the patient and staff narratives told us stories of achievements and also uncovered challenges that we can solve to make patients' stays a little easier. A lot of patients told us they forgot their phone chargers. In the pre-COVID-19 era, that would be no problem - a friend or family member would just bring one by on their next visit. But now, family members can't just stop by. And 
those phones are more important than ever to help patients stay connected to and supported by their loved ones. So, we bought a bunch of phone chargers and made them available on every unit.

But it's the stories we hear from patients and staff that keep us committed. Nurses and other team members tell us they're grateful to see patients connecting, smiling, and comfortable when we call. It eases the burden of their responsibility to care for patients through layers of PPE and without some of the simple comforts of touch and visible smiles that were staples of their pre-COVID care. We know that kind of support is healing.

Patients tell us they feel guilty seeing staff enter in PPE, fearing they will infect their care team members. During our calls, we are able to reassure them that our care team members are committed to caring and that our PPE precautions are keeping them safe. We know those reassurances help patients start their emotional recovery before they even leave.

Patients also tell us such amazing stories of care and compassion from our nursing staff. We hear of simple comforts, such as nurses bringing water before the patient even has a chance to ask. We hear of nurses listening and providing reassurances as patients relay fears of giving birth during a pandemic. And we hear of teamwork, coordination, and the kind of professionalism that is the hallmark of every team member, from physicians and nurses to environmental services and phlebotomists. We are able to share those kudos to our team members and let them know how grateful our patients are for the care they're receiving. We know that the simple act of recognition is a kind of love.

\section{Measurable Outcomes \& Continued Practice}

UChicago Medicine has been collaborating with other institutions to understand trends that they are experiencing and ways to still serve our patients with innovation and care. Regarding the pandemic, most institutions witnessed a decrease in their outcomes on patient experience; yet UCM ended the year strong surpassing target. In fact, during the months in which virtual rounding was a part of our platform, we enjoyed a 5\% increase in outcomes for those patients who received a round compared to those months in which only Leader Rounds were a part of our patient experience repertoire.

The implications of the patient experience improvement data convinced our team of the importance to continue to bring this new implementation of rounding processes and practical changes to our patient experience arsenal.

Embedded in our continuous improvement practices, this data will be continued to be monitored, as well as acted upon, to continue to share a culture of experience excellence.

\section{Creating a Meaningful Human Experience}

It would have been easy to look at this pandemic and say, "There's nothing we can do about patient and team member experience right now. And it doesn't matter as much as infection prevention anyway." But that would not have been true to the core mission and beliefs that live at the heart of our PEEP team. We know that love heals hearts. We know that caring eases minds and bodies. And we know that connection soothes the soul.

I am so proud of everything our PEEP team has done to rise to the challenges of COVID-19. The human experience is more important now than ever before. Because of the creativity and innovation that came from this pandemic, we as an institution are continuing our virtual care call connections program and embedding our patient experience team support in all that we do and who we are. I'm grateful that the University of Chicago Medicine continues to serve our community with humanity. 